\title{
L183 (L134N) Revisited
}

\section{The dust content ${ }^{\star}$}

\author{
L. Pagani ${ }^{1}$, A. Bacmann ${ }^{2}$, F. Motte ${ }^{3}$, L. Cambrésy ${ }^{4}$, M. Fich ${ }^{5}$, G. Lagache ${ }^{6}$, M.-A. Miville-Deschênes ${ }^{6,7}$, \\ J.-R. Pardo ${ }^{8}$, and A. J. Apponi ${ }^{9}$ \\ 1 LERMA \& UMR 8112 du CNRS, Observatoire de Paris, 61 Av. de l'Observatoire, 75014 Paris, France \\ 2 ESO, Karl-Schwarzschild-Str. 2, 85748 Garching bei München, Germany \\ 3 CEA, DSM, DAPNIA, Service d'Astrophysique, CE Saclay, 91191 Gif-sur-Yvette Cedex, France \\ 4 Observatoire Astronomique de Strasbourg, UMR 7550, Université Louis Pasteur, 67000 Strasbourg, France \\ 5 Physics Department, University of Waterloo, Waterloo, Ontario, N2L 3G1, Canada \\ ${ }^{6}$ IAS, Bât. 121, Université Paris-Sud, 91435 Orsay Cedex, France \\ 7 CITA, University of Toronto, 60 St. George Street, Toronto, Ontario, M5S 3H8, Canada \\ 8 Instituto de Estructura de la Materia, Dpto de Astrofísica Molecular e Infrarroja CSIC, Madrid, Spain \\ 9 University of Arizona, Steward Observatory, 933 N. Cherry Ave., Tucson, AZ 85721, USA
}

Received 16 July 2003 / Accepted 12 November 2003

\begin{abstract}
We present here a complete dust map of $\mathrm{L} 183(=\mathrm{L} 134 \mathrm{~N})$ with opacities ranging from $A_{\mathrm{V}}=3$ to 150 mag. Five peaks are identified as being related to known molecular peaks and among these dust peaks two are liable to form stars. The main peak is a prestellar core with a density profile proportional to $r^{-1}$ up to a radius of $\sim 4500 \mathrm{AU}$ and the northern peak could possibly be on its way to form a prestellar core. If true, this is the first example of the intermediate steps between cloud cores and prestellar cores during the quasi-static contraction. Additionally, the low dust temperature of the core reported in Pagani et al. (2003b) is confirmed, and the ISOPHOT data are shown to be inappropriate for finding such cores. In the inner core, $T_{\text {dust }} \approx 7.5 \mathrm{~K}$ and could be as low as $6.7 \mathrm{~K}$.
\end{abstract}

Key words. ISM: abundances - ISM: molecules - ISM: dust, extinction - ISM: structure - ISM: individual: L134N ISM: individual: L183

\section{Introduction}

In recent years, with the advent of various dust detectors in the near infrared (NIR), far infrared (FIR), millimeter ( $\mathrm{mm}$ ) and sub-millimeter (submm), it has become clear that dust studies are the only way to evaluate the mass of cold clouds; gas tracers such as $\mathrm{C}^{18} \mathrm{O}$ being unreliable because of strong depletion effects. For the same reason, prestellar cores, suffering from high depletion of molecules, are barely detectable via molecular studies and only dust mapping has been able to reveal these cores.

Since dust is optically thin in most of the submm and far infrared regime, it is in principle easy to analyze in terms of radiative transfer. The lack of velocity signature, however, makes it

Send offprint requests to: L. Pagani,

e-mail: laurent.pagani@obspm.fr

* Based on observations made with the CFHT, the Iram-30 m and ISO, an ESA project with instruments funded by ESA Member States (especially the PI countries: France, Germany, The Netherlands and the UK) with the participation of ISAS and NASA. difficult to separate emission from unrelated molecular clouds on the same line of sight or even to separate different components within the same cloud. Another difficulty is that the dust emitters (the grains) vary in size and type and thus their emitting properties vary as discussed theoretically by Hildebrand (1983), Draine \& Lee (1984) or Rengarajan (1984) and recently demonstrated by Dupac et al. (2003). Radial variations across low extinction clouds have been recently shown by Stepnik et al. (2003) and Cambrésy et al. (2001). Dust in emission is thus somewhat difficult to use to measure total column density and special care must be taken in its interpretation. A better way to estimate dust column density is to estimate dust absorption in the near infrared as shown by Lada et al. (1994, 1999). It is considered that the absorptivity of the dust in the NIR changes very little with grain size (Mathis 1990) and thus it is an excellent way to measure dust column density and hence total gas+dust column density, i.e. assuming the gas-to-dust ratio of $\sim 100$ holds.

L183, also known as L134N but with slightly different coordinates, starless, high above the galactic plane $\left(b=+37^{\circ}\right)$ 
and close to us, is ideally situated for detailed studies of a cold dark cloud. Its high galactic latitude eliminates pollution by other cloud emission (especially in continuum), and its proximity $(110 \pm 10 \mathrm{pc}$, Franco 1989) provides a high linear resolution $\left(1^{\prime \prime}=110 \mathrm{AU}\right)$. In previous studies, a $15 \mathrm{~K}$ dust region has been detected based on IRAS FIR observations (Laureijs et al. 1991, 1995), and colder dust peaks were identified at $\lambda=$ $0.85 \mathrm{~mm}$ with JCMT bolometers (Ward-Thompson et al. 1994, 2000). Based on these data and ISOPHOT maps, Lehtinen et al. (2003) present four cold condensations. However we cannot confirm the existence of all of their sources as demonstrated by Pagani et al. (2003b, hereafter Paper I).

This paper, the second of a series on this source (Paper I; see also Pagani et al. 2002, 2003a), presents the dust studies we have made both in absorption and in emission with data ranging from $1 \mu \mathrm{m}$ to $1.2 \mathrm{~mm}$ in wavelength. We present the observations in Sect. 2 and discuss the results in Sect. 3. Mass, interesting dust peaks and dust temperature are discussed in Sect. 4 and we present our conclusions in Sect. 5.

\section{Observations}

$I, H$ and $K^{\prime}$ band observations were performed at CFHT. ISOCAM and ISOPHOT data were retrieved from the ISO Data Archive $^{1}$ and MAMBO $1.2 \mathrm{~mm}$ observations were performed at the IRAM 30-m radiotelescope.

The CFHT has been used with both the CFHT12K and the CFHTIR cameras. In the Service Observing Queue mode, a deep $I$ band, large scale $\left(12000 \times 8000\right.$ pixels covering $\left.42^{\prime} \times 28^{\prime}\right)$ image of the cloud was obtained with the CFHT12K in April 2002. This image covers the whole cloud at once and presents dust-free pieces of sky in the corners which were used to set our zero extinction level. The data were taken in 7 individual exposures, one short (30 s), six long (about $10 \mathrm{~min}$ each). The seeing was very low $\left(0.5-0.6^{\prime \prime}\right)$, but the night was not photometric. The poor weather, however, did not affect our ability to measure the extinction in the outskirts of the cloud because only a count of the number of stars in the field was needed to do so. The data reduction was performed with the TERAPIX tools by M. Dantel-Fort and SExtractor (Bertin \& Arnouts 1996) was used to build the source catalogue. The final image was calibrated with data from the DENIS survey (Epchtein 1997; Epchtein et al. 1999) which provided us with accurate positions and magnitudes down to $I=18 \mathrm{mag}$. The cross-identification of the CFHT12K data with DENIS yielded 574 matches within 2", and the CFHT12K catalogue was complete up to $I=23.6$ mag. Approximately 27000 objects were detected, yielding an average of 22 sources per square arcmin.

The CFHTIR camera $\left(1000 \times 1000\right.$ pixels covering $3.6^{\prime} \times$ $\left.3.6^{\prime}\right)$ was used with the near infrared (NIR) wide $H$ and $K^{\prime}$ filters to map the inner core of the cloud. The observations were conducted in June 2002 during 2.5 nights. The seeing was excellent at the beginning $\left(0.4-0.5^{\prime \prime}\right)$ only degrading slightly toward the end of the second night and at the end of third (half-)night. The sky was photometric and the calibration was performed with sources that were detected in the 2MASS

\footnotetext{
${ }^{1}$ http://www.iso.vilspa.esa.es/ida/index.html
}

survey (Cutri et al. 2000). The cross-identification of the CFHIR data with 2MASS leads to 63 matches in $H$ (for $H<15.1$ ) and 47 matches in $K^{\prime}\left(K^{\prime}<14.3\right)$ for a $2^{\prime \prime}$ search radius. An $18^{\prime} \times 18^{\prime}$ region was mapped with 25 adjacent fields. The adjacent fields were overlapped slightly by using the shift+add observing method. To concentrate as much as possible on the most extincted inner core, we spent only 2 min per field on the outskirts for both filters and concentrated on the 9 inner fields, with particular emphasis on the two fields covering the peak and the ridge (Paper I) where several hours were spent per filter and per field. A rather uniform coverage of the cloud was obtained with a similar number of detected sources per frame. There are typically 40 sources per field which yields an average of 3.2 sources per square arcmin. The weakest sources detected with both the $H$ and $K^{\prime}$ filters reach $\left(H, K^{\prime}\right)=(21.3,20.6)$ mag and $\left(H, K^{\prime}\right)=(21.9,20.2) \mathrm{mag}$. With a positional error smaller than $1^{\prime \prime}$ and a photometric accuracy of about 5\%, DENIS and 2MASS are excellent substitutes to calibration fields.

The LW2 (5.0-8.5 $\mu \mathrm{m}$ filter) ISOCAM map covers $5^{\prime} \times$ $5^{\prime}$ with $6^{\prime \prime} \times 6^{\prime \prime}$ pixels. The data was reduced following the method described in Miville-Deschênes et al. (2000). Thanks to the data redundancy and oversampling, the final map can be presented with pixels of $3^{\prime \prime} \times 3^{\prime \prime}$. The rms noise in the central part of the map is $0.005 \mathrm{MJy} \mathrm{sr}^{-1}$. A bright star detected in the upper right corner of the map and recorded in the 2MASS database allowed us to correct the pointing to better than $1^{\prime \prime}$. The error was $(\Delta \alpha, \Delta \delta)=\left(4^{\prime \prime}, 3^{\prime \prime}\right)$.

The ISOPHOT data represent two $30^{\prime} \times 30^{\prime}$ maps at 100 and $200 \mu \mathrm{m}$ (see Lehtinen et al. 2003) and a series of declination strips from 100 to $200 \mu \mathrm{m}$ (see Ward-Thompson et al. 2002; Lehtinen et al. 2003). They were discussed in detail in Paper I where it is shown that only the two maps are usable. Therefore, only our own reduction of the $200 \mu \mathrm{m}$ map obtained with the latest version of the PIA reduction package (PIA V10.0) was used. To eliminate the diffuse dust emission contribution, it is not sufficient to subtract a constant value to bring to zero the ISOPHOT maps in the upper right corner where only diffuse dust emission is present. An inclined plane fitted to the IRAS $60 \mu \mathrm{m}$ emission slope must also be subtracted. In the following, only the molecular cloud cold dust $200 \mu \mathrm{m}$ emission will be considered.

The MAMBO (IRAM 30-m bolometer at $1.2 \mathrm{~mm}, 11^{\prime \prime}$ resolution) data were taken in December 2001 and February 2002. Opacity was in the range $0.1-0.3$ during the observations. We made multiple fast scans of a large area $\left(\sim 15^{\prime} \times 15^{\prime}\right)$. The observational details are discussed in Paper I. The data have been reduced with NIC, the IRAM bolometer package (Broguière et al. 1995) and a skynoise reduction technique described in Motte et al. (in preparation). The final rms is $0.8 \mathrm{MJy} / \mathrm{sr}$ on the map smoothed to $15^{\prime \prime}$ resolution. Systematic errors in the baseline level might be larger than that because large scale $\left(>15^{\prime}\right)$ features of the map are lost due to the dual-beam mapping and reduction techniques and because we have not reached the borders of the cloud, especially in the southern part. We have however estimated that the peak emission cannot be underestimated by more than $2 \mathrm{MJy} / \mathrm{sr}$. Another reason limiting the quality of the data arises from a non-Gaussian electronic noise in the receiver during the 2001-2002 winter season. For the off peak 


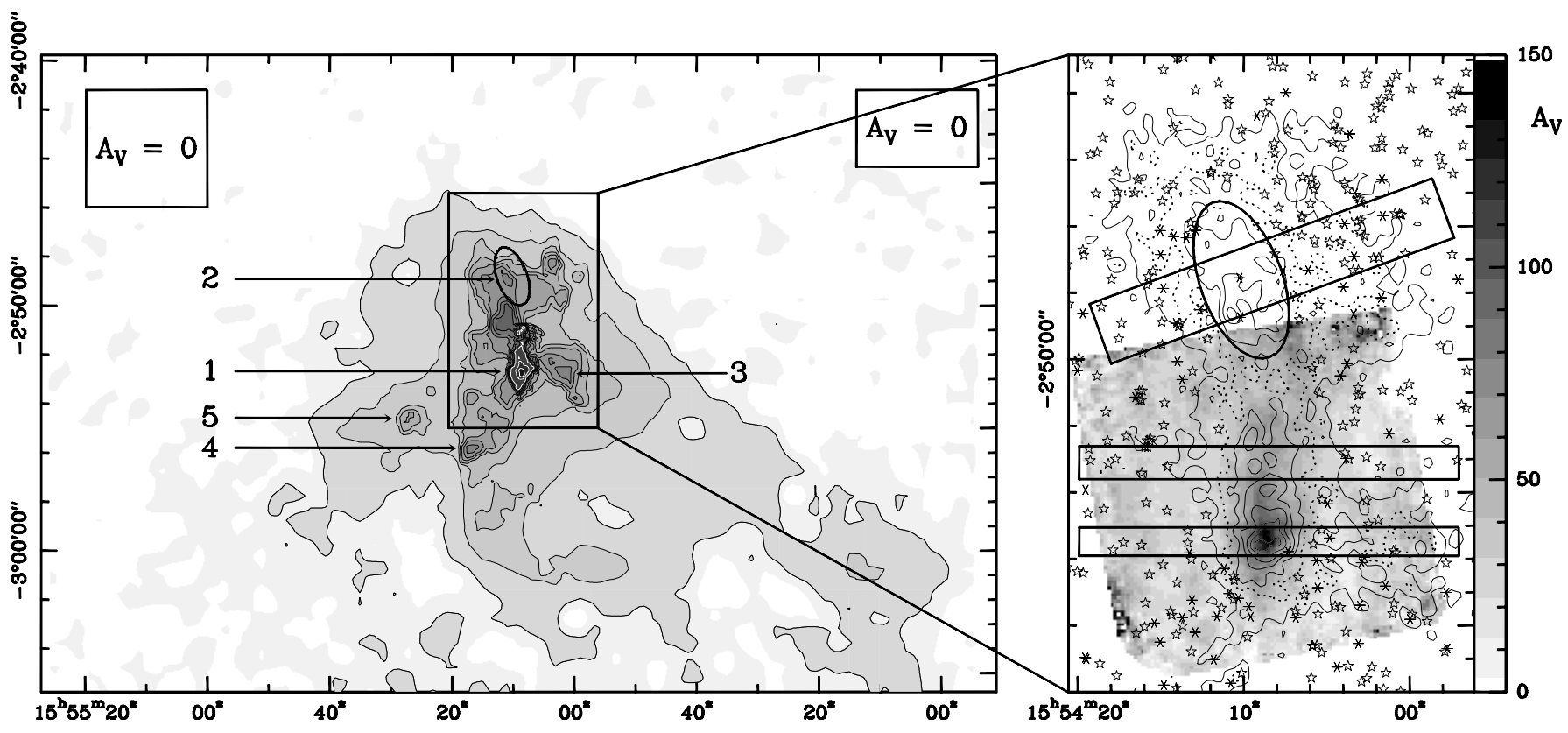

Fig. 1. The composite extinction image of the whole L183 cloud. This is the combination of the $I, H K^{\prime}$ and ISOCAM extinction maps all converted to the visual extinction scale, $A_{\mathrm{V}}$ (mag). Levels are $A_{\mathrm{V}}=5$ to 50 by 5 (black contours) and 60 to 120 by 30 (white contours) mag. The pale shade of grey is set at $A_{\mathrm{V}}=3 \mathrm{mag}$. The two boxes in the upper corners indicate where the zero magnitude extinction has been set. The numbers indicate the peaks which have a molecular counterpart. The blow-up region shows the sole ISOCAM extinction map (grey scale) with the $1.2 \mathrm{~mm}$ dust emission superposed to it (contours from 7 to 22 by $2.5 \mathrm{MJy} / \mathrm{sr}$ ). The $9.5 \mathrm{MJy} / \mathrm{sr}$ contour is dotted. Sources detected in both $H$ and $K^{\prime}$ are indicated as stars, sources detected only in $K^{\prime}$ as asterisks. There is no available extinction measurement inside the ellipse and only lower limits can be given. Boxes in the blow-up region delineate the location and the width of the strips shown in Fig. 4.

emission, the relative flux uncertainty was conservatively estimated to reach as high as $40 \%$. For the peak emission, the absolute calibration uncertainty was estimated to be about $20 \%$.

\section{Results}

The range of dust column densities make it impossible for any present single instrument to record all the dust content in this cloud at once. Thus it was necessary to combine 3 different sets of data to obtain the complete dust map of L183 (Fig. 1): the $I$ band accounts for low and intermediate extinction $\left(A_{\mathrm{V}}<15\right)$, $H-K^{\prime}$ color excess for high extinction $\left(15<A_{\mathrm{V}}<30\right)$ and ISOCAM $7 \mu \mathrm{m}$ for very high extinction. The following discussion reports extinction measurements converted to a common visual extinction scale $\left(A_{\mathrm{V}}\right)$ although the dust properties in the $V$ band may change drastically in very dense and opaque cloud cores and thus the values we give may not reflect the actual $V$ band extinction. A more reliable estimate is the $K$ band extinction $\left(A_{K}\right)$ as the NIR dust properties are considered to be more stable (Mathis 1990). It can be obtained by multiplying all $A_{\mathrm{V}}$ values by $0.113 . I$ and $H-K^{\prime}$ extinction measurements are comparable in the range 5-15 mag. The $I$ band extinction measurement shows clearly a saturation above $15 \mathrm{mag}$ and the $H-K^{\prime}$ color excess becomes insensitive below 5 mag of extinction given the star density and the spatial resolution we chose (and the $H-K^{\prime}$ map does not cover all the cloud anyway). $H-K^{\prime}$ extinction measurements do not go beyond $\sim 40 \mathrm{mag}$ owing to a lack of bright sources behind the cloud, which results from the cloud being high above the galactic plane.
The star count and the color excess methods are described in Cambrésy et al. (2002). Adaptive cell sizes are used in order to optimize the spatial resolution and to limit the non-linearity effect inherent to star counts and reddening methods. No foreground star correction is required for L183, which is located at high galactic latitude and close to us.

The source counts convert to visual extinction as follows:

$A_{\mathrm{V}}=\left(\frac{1}{a} \log \frac{1}{D}\right) \times\left(\frac{A_{I}}{A_{\mathrm{V}}}\right)^{-1}+\mathcal{Z}_{\mathrm{cnt}}$

where $a=0.21$ is the slope of the luminosity function (restricted to the completeness limit $I<23.6 \mathrm{mag}$ ), $D$ the source density in each cell (chosen to contain 10 sources), $A_{I} / A_{\mathrm{V}}=$ 0.482 the conversion factor from the extinction law (Rieke \& Lebofsky 1985) and $\mathcal{Z}_{\text {cnt }}$ the zero point for the extinction map.

The relation for the $H-K^{\prime}$ color excess is simply:

$A_{\mathrm{V}}=\left(\frac{A_{H}}{A_{\mathrm{V}}}-\frac{A_{K^{\prime}}}{A_{\mathrm{V}}}\right)^{-1} \times\left(H-K^{\prime}\right)+\mathcal{Z}_{\mathrm{col}}$

where $\left(A_{H} / A_{\mathrm{V}}-A_{K^{\prime}} / A_{\mathrm{V}}\right)^{-1}=15.87$, and $\mathcal{Z}_{\text {col }}$ is the zero point. The number of objects per cell is fixed to 3 for the reddening method.

The zero points are set assuming there is no extinction at the edges of the $I$ band field (see Fig. 1). This assumption is confirmed by a larger scale analysis $(2 \times 2 \mathrm{deg})$ of 2 MASS and $\mathrm{HI}$ data. The latter indicates that in a pixel $40^{\prime}$ diameter centered on the cloud, the average HI column density is $8.5 \times$ $10^{20} \mathrm{~cm}^{-2}$, translating to $A_{\mathrm{V}} \approx 0.4$ mag which is well within the noise of the $I$ band measurement (using the standard conversion formula $N(\mathrm{HI}) \simeq 2 \times 10^{21} \times A_{\mathrm{V}} \mathrm{cm}^{-2}$, Bohlin et al. 1978). 
The ISOCAM $7 \mu \mathrm{m}$ absorption map has been converted to a visible extinction map using the method described by Bacmann et al. (2000) except that the conversion was done using the surrounding $H-K^{\prime}$ infrared excess map instead of using dust emission and $\mathrm{C}^{18} \mathrm{O}$ measurements. The result is thus independent of the actual dust temperature and of possible CO depletion. Two regions (outside the main peak for which no stars were seen in deep $K^{\prime}$ images) have been used to do the conversion. Those two regions were chosen around the offsets $\left(-100^{\prime \prime}, 0\right)$ and $\left(-50^{\prime \prime},-50^{\prime \prime}\right)$ with respect to the peak. This method implies that the features seen in the image are due to the absorption from the core only, and not to structure in the mid-infrared background or foreground emission. Although, the statistical uncertainty on the calibration is about $7 \%$, the global conversion suffers from critical estimates of the foreground and background infrared emission, which are not well known (see Bacmann et al. 2000). Hence, the total uncertainty on the absolute extinction is substantially higher, reaching $+66 /-33 \%$ at the peak $\left(A_{\mathrm{V}} \approx 150 \mathrm{mag}\right)$.

The spatial resolution varies within the final map (Fig. 1) from $1^{\prime}$ to $5^{\prime}$ for the $I$ band counts, $\sim 1^{\prime}$ for the $H-K^{\prime}$ reddening zone and $6^{\prime \prime}$ for ISOCAM. The central part of the map (the main core and the northern ridge), which is also discussed in Paper I, is expanded in Fig. 1 (and the peak alone in Fig. 2). Shown in this zoom image are the ISOCAM dust extinction map (pixels), the MAMBO $1.2 \mathrm{~mm}$ emission map (contours) and sources detected in both $H$ and $K^{\prime}$ (marked as stars) or only in $K^{\prime}$ (marked as asterisks). As noted in Paper I the agreement between ISOCAM and MAMBO is excellent. Furthermore, the NIR sources are all outside the same MAMBO brightness contour, the dotted one, which corresponds to $B(1.2 \mathrm{~mm})=$ 9.5 MJy/sr. The absence of NIR sources inside this contour indicates that the optical extinction reaches at least 40 mag. This is consistent with the measured dust emission if we set $T_{\text {dust }} \approx 8 \mathrm{~K}$ with a dust opacity $\kappa_{1200 \mu \mathrm{m}}=0.009 \mathrm{~cm}^{2} \mathrm{~g}^{-1}$ (as briefly discussed in Paper I) in the following equation

$B(v)=B_{v}\left(T_{\text {dust }}\right) \times \tau(v) \mathrm{MJy} / \mathrm{sr}$

where $B_{v}\left(T_{\text {dust }}\right)$ is the Planck function, $\tau(v)$ the dust opacity at frequency $v$.

$B(v)=\frac{h v^{3}}{c^{2}} \frac{1}{\exp \left(\frac{h v}{k T}\right)-1}\left(\frac{\tau(v)}{A_{\mathrm{V}}}\right) A_{\mathrm{V}} \quad \mathrm{MJy} / \mathrm{sr}$

where $h, k$ and $c$ have their usual meaning and $\frac{\tau(v)}{A_{\mathrm{V}}}$ represents the dust opacity coefficient at frequency $v$.

$\frac{\tau(v)}{A_{\mathrm{V}}}=\frac{\tau(v)}{N_{\mathrm{H}_{2}}} \frac{N_{\mathrm{H}_{2}}}{A_{\mathrm{V}}}$

and

$\frac{\tau(v)}{N_{\mathrm{H}_{2}}}=\mu m_{\mathrm{H}} \kappa_{v}$

$\frac{N_{\mathrm{H}_{2}}}{A_{\mathrm{V}}}=10^{21} \mathrm{~cm}^{-2} \mathrm{mag}^{-1}$

with the mean molecular weight $\mu=2.33$, the atomic hydrogen mass $m_{\mathrm{H}}=1.67 \times 10^{-24} \mathrm{~g}$ and $\kappa_{v}$ is the dust opacity per unit

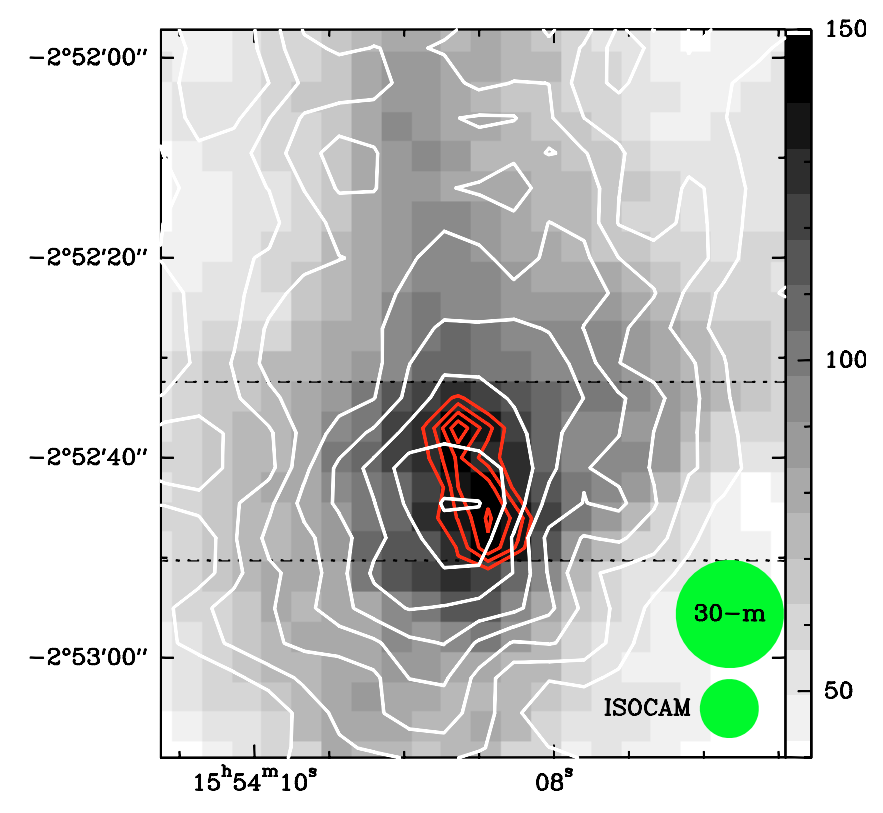

Fig. 2. Peak dust extinction (grey scale and contours) and emission (white contours) in the cloud. Grey extinction contours represent $A_{\mathrm{V}}=$ 130 to 145 by 5 mag. White contours represent $1.2 \mathrm{~mm}$ dust emission. The two horizontal dot-dashed lines delineate the pixel lines which have been averaged together to analyze the peak profile (Fig. 3).

mass column density at frequency $v$ (though $\kappa$ subscript is generally given in wavelength). Equation (7) comes from Bohlin et al. (1978). A discussion and a thorough modeling of $\kappa_{v}$ can be found in Motte et al. (1998) and Ossenkopf (1993), respectively. This result ( $T_{\text {dust }} \approx 8 \mathrm{~K}$ ) is consistent with the ridge temperature determined from ISOCAM and ISOPHOT measurements in Paper I.

Finally, the ellipse traced in Fig. 1 encompasses the only region of the cloud that has only lower extinction limits. There are no NIR $H$ band sources inside this ellipse and the ISOCAM map did not cover this region.

\section{Discussion}

\subsection{The cloud dust mass}

The total mass of the cloud depends somewhat on the chosen extinction limits. Under $A_{\mathrm{V}}=5$ mag, L183 merges with L134 $\left(\sim 2^{\circ}\right.$ further south) and the cloud mass is then the mass of the whole complex. Limiting ourselves to the full image presented here, we find a total mass of $78 M_{\odot}$ and $56 M_{\odot}$ above $A_{\mathrm{V}}=$ 5 mag of which only $50 M_{\odot}$ belong to the main cloud.

\subsection{The pre-stellar core and other peaks}

The main map contains several peaks (Fig. 1). Each peak that has a molecular peak counterpart in their vicinity is numbered in the figure. Peak 1 (the pre-stellar core) is close to the molecular peak C described by Dickens et al. (2000). Likewise, Peak 2 is close to N, Peak 3 is close to W, and Peak 4 is close to $S$, where all letters come from Dickens et al. (2000). The weakest of these peaks (Peak 5) has only $A_{\mathrm{V}}=20-25$ mag but is 
coincident with the $100 / 200 \mu \mathrm{m}$ Eastern peak seen in the ISOPHOT maps at $\alpha_{2000}=15 \mathrm{~h} 54 \mathrm{~m} 25.8 \mathrm{~s}, \quad \delta_{2000}=$ $-2^{\circ} 54^{\prime} 36.5^{\prime \prime}$ (Fig. 5, Lehtinen et al. 2003) and with our $\mathrm{C}^{18} \mathrm{O}$ and $\mathrm{C}^{17} \mathrm{O}$ peak (Pagani et al. 2002). We will call it Peak E, for east, as a molecular peak, for consistency with Dickens et al. (2000). Thanks to the good astrometry of the ISOCAM image, Peak 1 is unquestionably 30" south of molecular Peak C, but the relative position of the other peaks is less sure because they are derived from a limited number of NIR sources and their position is only known to $\sim 30-60^{\prime \prime}$. Peaks 3 to 5 will not be discussed further.

\subsubsection{Peak 1}

Figure 2 shows an enlargement of Peak 1. The highest opacity contours of the ISOCAM data are shown as grey contours to emphasize the elongated feature. This feature, oriented northsouth, is almost $20^{\prime \prime}$ in length at $A_{\mathrm{V}}=130$ mag and remains unresolved in width (i.e. horizontally) with possibly two subpeaks inside. The whole peak itself is at the southern end of a long ridge $6^{\prime}$ in length also oriented North-South (see Fig. 1 and Paper I). Though the contrast between the two sub-peaks and the dip that lay between is less than $1 \sigma$, they possibly indicate the presence of 2 pre-stellar seeds separated by $9^{\prime \prime}\left(=10^{3} \mathrm{AU}\right)$. Because the profiles of the two peaks are similar, they were averaged to reduce the noise, i.e. the 6 horizontal pixel lines delineated in Fig. 2. Because the northern sub-core is one pixel further to the east, the 3 lines of this sub-core were shifted by one pixel to the west so that both sub-core peaks were aligned before averaging. Both sides were then folded together symmetrically and summed. In the resulting image, an $r^{-1}$ density variation was found from $6^{\prime \prime}$ away from the center up to $40^{\prime \prime}$ away (Fig. 3), that is a radius of $\sim 4500$ AU Such a density variation has not been seen in other ISOCAM density profile studies (Bacmann et al. 2000), but has been theoretically investigated by McLaughlin \& Pudritz $(1996,1997)$ and is known as a logotropic profile. The density inside the central $9^{\prime \prime}$ radius hardly varies and reaches $2 \times 10^{6} \mathrm{~cm}^{-3}$. The corresponding mass is less than $0.01 M_{\odot}$ for each of the two subcores, $0.06 M_{\odot}$ for the whole elongated peak holding the subcores assuming it is cylindrical. The total mass of the core in the $1^{\prime}$ radius (the limit where the core seems to decouple from the ambient gas as suggested by the ISOCAM profile) is $\approx 2.5 M_{\odot}$.

\subsubsection{Peak 2}

The northern peak is delimited by the $148^{\prime \prime} \times 74^{\prime \prime}$ ellipse traced in Fig. 1. Interestingly, on the main map, the ellipse is seen to overlap a large opacity gradient, starting at $A_{\mathrm{V}} \approx 15 \mathrm{mag}$ at the northern tip of the ellipse up to 35 mag at the southern tip. This gradient arises from the relatively low NIR excess detected in the sources north and west of the ellipse and indicates that the dust column density gradient must be very steep on the northwest side of the ellipse to reach at least 40 mag of visual extinction. This gradient could simply be caused by a lack of sources behind this part of the cloud, but this is probably not the case. Very few sources have been found inside the ellipse, but

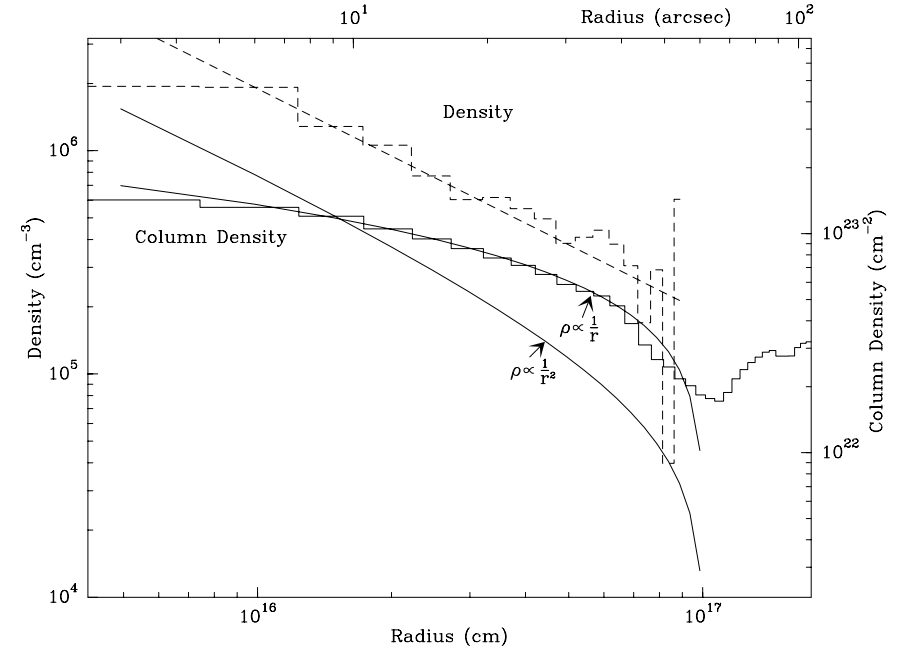

Fig. 3. Radial volume density and column density fits. Column density quantities are represented by full lines while volume density quantities are represented by dashed lines. Data are displayed as histograms while fits are displayed as curves. The radial density profile is obtained by decomposition of the column density profile in onion layers. Continuous curves represent the $r^{-1}$ fit to the density profile and the expected column density profile obtained from a $r^{-1}$ and a $r^{-2}$ density profiles. The fits offsets are adjusted to fit the 3rd pixel, $9^{\prime \prime}$ away $\left(\sim 1.5 \times 10^{16} \mathrm{~cm}\right)$ from center.

those found are only in $K^{\prime}$ and without any $H$ counterpart. All these sources have $K^{\prime} \geq 19.3 \mathrm{mag}$ and an extinction lower limit of $\sim 43$ mag for the brightest. Futhermore, the ellipse is coincident with the $1.2 \mathrm{~mm}$ emission contour at $12 \mathrm{MJy} / \mathrm{sr}$ which indicates $A_{\mathrm{V}} \geq 50 \mathrm{mag}$ in the same conditions as above (Sect. 3, Eqs. (3)-(7)).

The next $1.2 \mathrm{~mm}$ contour (14.5 MJy/sr) covers the southern part of the ellipse only and underlines the local peak (Peak 2 in the main map) with an equivalent dust extinction of $\sim 60 \mathrm{mag}$. The final contour (16.5 MJy/sr, $A_{\mathrm{V}} \approx 70 \mathrm{mag}$ ) possibly traces the center of that peak but such small peaks can also be remnants of the electronic noise. Interestingly enough, the total mass inside the ellipse is $0.69 M_{\odot}$ from the $1.2 \mathrm{~mm}$ emission (with $T_{\text {dust }}=8 \mathrm{~K}$ and $\kappa_{1200 \mu \mathrm{m}}=0.009 \mathrm{~cm}^{2} \mathrm{~g}^{-1}$ ) which is comparable to the mass we find $\left(0.77 M_{\odot}\right)$ in the same ellipse centered at $\alpha_{2000}=15 \mathrm{~h} 54 \mathrm{~m} 08.48 \mathrm{~s}, \delta_{2000}=-2^{\circ} 52^{\prime} 08^{\prime \prime}$ and encompassing the main peak and a part of the ridge.

\subsubsection{Witnessing the contraction of the two peaks?}

Three cuts were made across the two peaks in the MAMBO $1.2 \mathrm{~mm}$ emission map. One horizontal (east-west) cut across the Peak 1, one cut across the ridge just north of Peak 1 and parallel to the first cut and one cut across Peak 2, tilted by $20^{\circ}$ to stay perpendicular to the major axis of the peak (see Fig. 1). The result is displayed in Fig. 4. The remarkable feature is that going from Peak 2 to the ridge to Peak 1, one can see that the profile slopes get steeper and the tops get narrower and eventually much stronger. From the ISOCAM data, the main core has a density gradient proportional to $r^{-1}$; this means that the Ridge and Peak 2 have even more shallow gradients. 


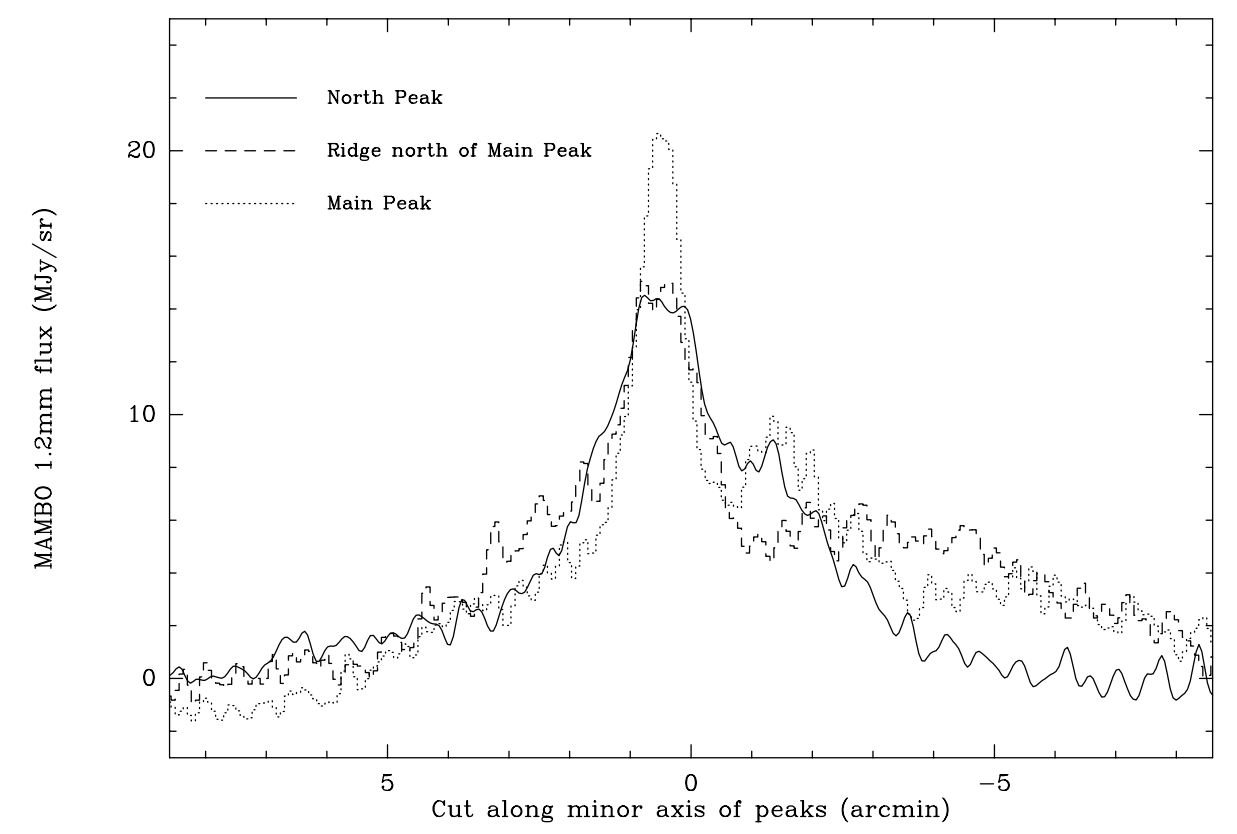

Fig. 4. 3 cuts across Peak 1 (main peak), the Ridge north of it and across Peak 2 (northern peak). The main peak cut also crosses Peak 3 (see Fig. 1).

One tentative explanation of these features (same amount of material, different peak height and width, evoluting profiles) is that we are witnessing the quasi-static contraction of the cores with the northern peak being younger (or less evolved) than the main peak and not yet condensed in a truly prestellar core. Dickens et al. (2000) suggest that the northern molecular Peak $(\mathrm{N})$ is younger than the other peaks (especially $\mathrm{C}$ and $\mathrm{W}$ ) because the $\mathrm{SO} / \mathrm{CS}$ ratio decreases from north to south. Although the use of this ratio as a chronometer is controversial because it also probably depends very much on initial conditions, it does seem to be coherent with our finding, however. Thus, if our interpretation is correct this would be the first observation of the intermediate steps transforming a cloud core into a prestellar core. It suggests that a noticeable gradient appears at the outskirts of the future core first, decoupling the core from the cloud before the core itself starts to collapse. It also suggests that the density gradient varies from zero (uniform density) to $r^{-1}$ during the contraction. We can wonder whether it keeps steepening to reach $r^{-2}$ which is found in other prestellar cores (Bacmann et al. 2000). Fast collapse would then take place. This result has some common features with the work done by Tafalla et al. (1998) and Williams et al. (1999) towards L1544 for which they found results inconsistent with the inside-out collapse model (Shu 1977). Interestingly, Peak 2 shows hints of a future prestellar core at its southern edge (highest MAMBO contours) just like Peak 1 formation at the southern edge of the Ridge. We have no explanation for this, but it indicates that the prestellar core does not have to form in the center of the parent cloud core.

\subsection{The dust temperature}

In the following discussion, the ISOPHOT map at $200 \mu \mathrm{m}$ represents the dust emission from the cloud alone and similarly $A_{\mathrm{V}}$ is measured above the diffuse dust extinction set to zero as explained in Sect. 2.

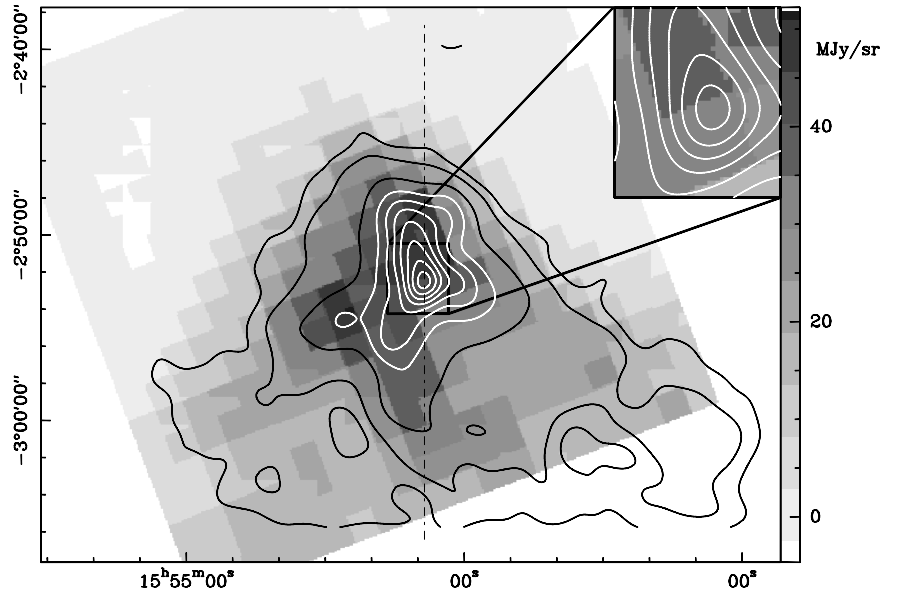

Fig. 5. ISOPHOT $200 \mu \mathrm{m}$ cold dust emission with visual extinction contours on top smoothed to $90^{\prime \prime}$. Contour levels are $A_{\mathrm{V}}=2.5$ and 5 to 45 by 5 mag. For low extinction levels, the correlation between the two maps is excellent but the core enlargement shows that the dust peak is not visible at all in the $200 \mu \mathrm{m}$ emission. The vertical dot-dash line shows the place of the cut displayed in Fig. 6.

In Paper I we presented some arguments showing that the dust core in this cloud is below $8 \mathrm{~K}$; a brief summary of that discussion follows. The dust extinction map, once smoothed to the ISOPHOT $200 \mu \mathrm{m}$ map resolution, still showed the main peak (Peak 1) while nothing appeared in the ISOPHOT map, which was in fact $10 \%$ below its maximum brightness at that position (see Figs. 5 and 6). The upper limit we found was based on a dust cloud analytical model (Zucconi et al. 2001) which we used to evaluate the amount of cold dust which emits at $100 \mu \mathrm{m}$ and evaluated its contribution to the $200 \mu \mathrm{m}$ emission. The remaining dust, too cold to emit at $100 \mu \mathrm{m}$, was subsequently estimated to be at $7.6 \pm 0.5 \mathrm{~K}$ for the main peak and at $8.0 \pm 0.5 \mathrm{~K}$ for the ridge. One limitation of the method was due to the important flat-field error (8 MJy/sr at $200 \mu \mathrm{m}$, Paper I). 


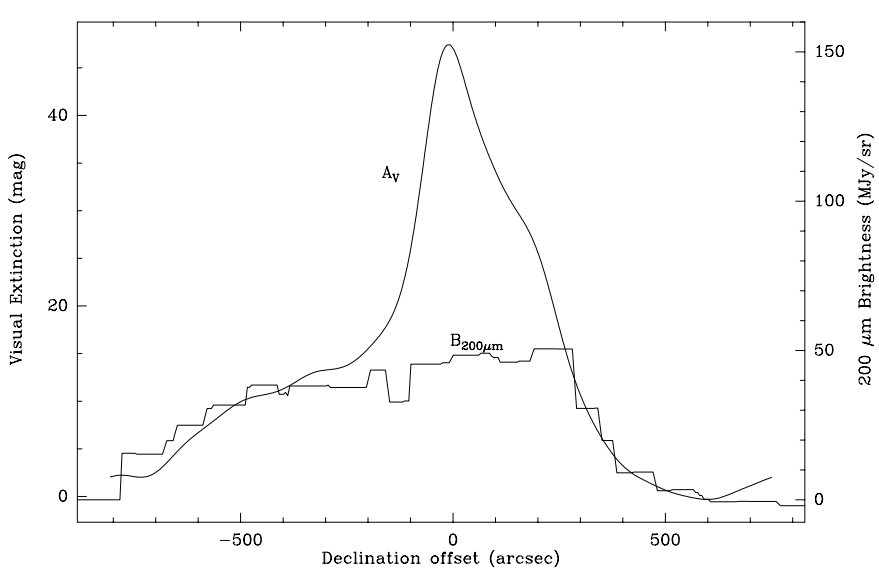

Fig. 6. ISOPHOT $200 \mu \mathrm{m}$ cold dust emission and visual extinction (smoothed to $90^{\prime \prime}$ ) cuts through Peak 1 (cut location is shown in Fig. 5). The dust peak is not visible at all in the $200 \mu \mathrm{m}$ emission. Left is south, right is north.

Here, the map shown in Fig. 5 was converted into a crosscorrelation map after reprojecting both sets of data on the same horizontal grid (Fig. 7). Because the cloud is optically thin at $200 \mu \mathrm{m}$ and because the visual extinction at the center of the cloud is only half the total extinction on the line of sight, both scales were divided by a factor of 2 to discuss the decrease of temperature towards the center of the cloud. This implies that the temperature variation is symmetrical on both sides (forefront and backside), i.e. that the Interstellar Radiation Field (ISRF) is essentially the same on both sides and that the temperature variation is mostly dependent upon the radial extinction profile. Figures 6 and 7 clearly show that the $200 \mu \mathrm{m}$ brightness does not increase beyond $A_{\mathrm{V}}$ (radial) $=$ $7.5 \mathrm{mag}$ (15 mag total). In particular, the dust peak is spectacularly missing in Fig. 6. Again, the simplest explanation is that the temperature decreases with increasing $A_{\mathrm{V}}$, as modeled by Zucconi et al. (2001), Evans et al. (2001) and Stamatellos \& Whitworth (2003). Indeed, below $15 \mathrm{~K}$, dust emission at $200 \mu \mathrm{m}$ is situated in the blackbody Wien regime and thus a small temperature variation has more impact on the emitted flux than a large variation in the dust column density as shown in Paper I. As a result, the ISOPHOT $200 \mu \mathrm{m}$ map does not trace Peak 1 at all, even though it does trace the large cold core in the cloud, when compared to its $100 \mu \mathrm{m}$ map counterpart (see Pagani et al. 2002, Juvela et al. 2002). The conclusion is that ISOPHOT data at $200 \mu \mathrm{m}$ are not well suited to find the very cold dust peaks inside cold cores, the external core layers being the major contributors to the $200 \mu \mathrm{m}$ emission. The effect is predicted to be still measurable at $450 \mu \mathrm{m}$ as discussed by Zucconi et al. (2001).

The dust temperature can now be re-estimated by a different method than in Paper I. Here, with the flat-field error being included in the data point spread, the temperature can be derived as a function of visual extinction statistically. From Eqs. (3) and (4), by summing the contribution of each dust layer at a different temperature, the integrated $200 \mu \mathrm{m}$ emission for

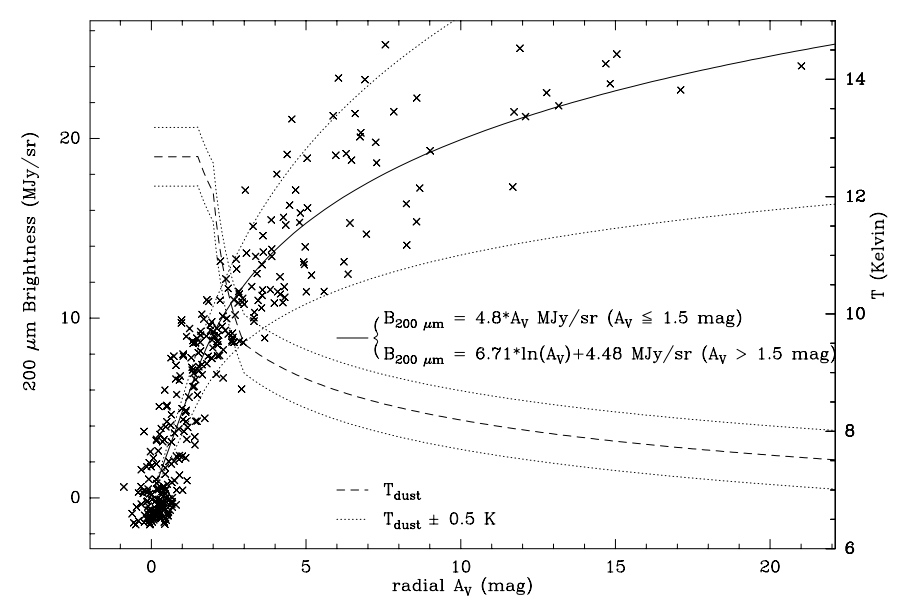

Fig. 7. ISOPHOT $200 \mu \mathrm{m}$ cloud emission cross-correlated with visual extinction smoothed to $90^{\prime \prime}$ and resultant temperature profile. This profile indicates the temperature for the given extinction layer, not the integrated temperature along the line-of-sight. The fitted temperature has been varied by $\pm 0.5 \mathrm{~K}$ and the $200 \mu \mathrm{m}$ brightness profile has been recomputed to estimate the robustess of the fit (dotted lines). In the Wien regime, $0.5 \mathrm{~K}$ represents a huge variation. The steep temperature drop between $A_{\mathrm{V}}=2$ and 3 mag is due to the 3 fold increase of the dust emissivity, going inwards.

a dust cloud of total opacity $A_{\mathrm{V}}$ along the line of sight is determined by

$B_{200 \mu \mathrm{m}}\left(A_{\mathrm{V}}\right)=2 \int_{0}^{\frac{A_{\mathrm{V}}}{2}} B_{v}\left(T_{\text {dust }}(x)\right) \frac{\tau_{v}}{x} \mathrm{~d} x \quad \mathrm{MJy} / \mathrm{sr}$.

If the cross-correlation data cloud can be fit with a function $F$ of the opacity

$B_{200 \mu \mathrm{m}}\left(A_{\mathrm{V}}\right)=F\left(A_{\mathrm{V}}\right) \mathrm{MJy} / \mathrm{sr}$

the result is

$B_{v}\left(T_{\text {dust }}\left(A_{\mathrm{V}}\right)\right) \propto \frac{\mathrm{d} F\left(A_{\mathrm{V}}\right)}{\mathrm{d} A_{\mathrm{V}}}$

where $\mathrm{B}_{v}$ is the Planck function at $v=1.5 \mathrm{THz}$ and assuming that $\tau_{v} / A_{\mathrm{V}}$ is essentially constant (see below). It appears in Fig. 7 that the best fit is obtained with

$B(200 \mu \mathrm{m})=4.8 \times A_{\mathrm{V}} \mathrm{MJy} / \mathrm{sr} \quad\left(A_{\mathrm{V}} \leq 1.5 \mathrm{mag}\right)$

$B(200 \mu \mathrm{m})=6.71 \times \ln \left(A_{\mathrm{V}}\right)+4.48 \mathrm{MJy} / \mathrm{sr} \quad\left(A_{\mathrm{V}}>1.5 \mathrm{mag}\right)$

and thus

$B_{v}\left(T_{\text {dust }}\left(A_{\mathrm{V}}\right)\right)=$ constant $\quad\left(A_{\mathrm{V}} \leq 1.5 \mathrm{mag}\right)$

$B_{v}\left(T_{\text {dust }}\left(A_{\mathrm{V}}\right)\right) \propto \frac{6.71}{A_{\mathrm{V}}} \quad\left(A_{\mathrm{V}}>1.5 \mathrm{mag}\right)$.

Finally, reversing the Planck function, one gets

$T_{\text {dust }}\left(A_{\mathrm{V}}\right)=12.7 \mathrm{~K} \quad\left(A_{\mathrm{V}} \leq 1.5 \mathrm{mag}\right)$

$T_{\text {dust }}\left(A_{\mathrm{V}}\right)=\frac{h v}{k} \frac{1}{\ln \left(1+\frac{h v^{3}}{c^{2}} \frac{A_{\mathrm{V}}}{6.71} \frac{\tau}{A_{\mathrm{V}}}\right)} \quad\left(A_{\mathrm{V}}>1.5 \mathrm{mag}\right)$

where $\frac{\tau}{A_{\mathrm{V}}}$ represents the dust opacity coefficient at $200 \mu \mathrm{m}$. It is now widely accepted that the submm and FIR dust emissivity increases from the diffuse to the dense medium as demonstrated by Stepnik et al. (2003). Though the cloud studied in 
their paper with a peak visual extinction of a few mag is not representative of our own case, we propose to put the diffuse to dense dust emissivity transition in the range $A_{\mathrm{V}}=2-3$ mag in our model, close to their own value of $A_{\mathrm{V}} \approx 2.1 \mathrm{mag}$. It is not yet clear whether the dust emissivity increase occurs at the same extinction depth for all clouds with similar external conditions (same ISRF) or if internal parameters are also important (such as depth at which depletion occurs, turbulence,...). Using

$$
\frac{\tau(200 \mu \mathrm{m})}{A_{\mathrm{V}}}=2.6 \times 10^{-4} \mathrm{mag}^{-1} \quad\left(A_{\mathrm{V}} \leq 2 \mathrm{mag}\right)
$$

for the diffuse dust and (after a linear increase from 2 to 3 mag)

$$
\frac{\tau(200 \mu \mathrm{m})}{A_{\mathrm{V}}}=8.8 \times 10^{-4} \mathrm{mag}^{-1} \quad\left(A_{\mathrm{V}} \geq 3 \mathrm{mag}\right)
$$

for the dense dust, we have retrieved the dust temperature from Eqs. (13). The dust profile is displayed in Fig. 7. We have then recomputed the $200 \mu \mathrm{m}$ brightness and checked that it follows the original fit. We find that the temperature profile starts at $\sim 13 \mathrm{~K}$ and goes down to $7.5 \mathrm{~K}$ at $A_{\mathrm{V}}$ (radial) $=21 \mathrm{mag}$ (or $42 \mathrm{mag}$ total). The diffuse to dense dust emissivity transition is revealed by the sudden drop in temperature between $A_{\mathrm{V}}$ (radial) $=2$ and $3 \mathrm{mag}$. This emissivity variation has not yet been introduced in the dust radiative transfer models (Zucconi et al. 2001, Evans et al. 2001, Stamatellos \& Whitworth 2003). It can be noted that $13 \mathrm{~K}$ is the value usually derived in other studies of dust in the molecular phase of the Interstellar Medium (see e.g. Bernard et al. 1999). Because we had to degrade the visual extinction data to the ISOPHOT C200 resolution $\left(\sim 90^{\prime \prime}\right)$, the peak extinction is only 42 mag instead of $\sim 150$ mag at the ISOCAM resolution ( $\left.6^{\prime \prime}\right)$. Thus, if the relation of Eq. (11) holds up to $A_{\mathrm{V}}$ (radial) $=75 \mathrm{mag}(150 \mathrm{mag}$ total $)$, then the core temperature in the deep center would only be $6.7 \mathrm{~K}$, similar to the model temperatures found by Zucconi et al. (2001) and Evans et al. (2001) in their cloud cores but below the new predictions made by Stamatellos \& Whitworth (2003) who predict that the core cannot drop in temperature below 7-8 K when the cloud embedding the core is taken into account in the model. We have also checked the sensitivity of the result on the variation of the emissivity and found that the dust temperature for the peak extinction would vary by $+0.6 /-0.5 \mathrm{~K}$ if we vary the dust emissivity by a factor 2 . Finally, we have varied the temperature profile by $\pm 0.5 \mathrm{~K}$ to check the dependency of the fit on the temperature (Fig. 7). It is clear that these variations encompass the possible temperatures which can fit our data.

\section{Conclusions}

A complete map of the dust content of the L183 cloud has been presented, yielding a total mass of $\gtrsim 78 M_{\odot}$. Among the many peaks which can be seen, 5 are remarkable because they are linked to the 5 known molecular peaks. The strongest peak is a prestellar core holding $2.5 M_{\odot}$ in a $1^{\prime}$ radius with a logotropic profile. Its temperature drops from 13 to $7.5 \mathrm{~K}$ and could be as low as $6.7 \mathrm{~K}$ in its deep center. These temperature variations are probably the reason why the ISOPHOT $200 \mu \mathrm{m}$ maps cannot show the very dense and cold cores inside clouds but only their immediate envelope. Thus great care should be taken when analyzing ISOPHOT data alone in such starless clouds. The northern peak, while having a similar mass on a similar surface than the central, main peak does not show a prestellar core but only strong column density variations at its edges. It could represent the intermediate step between a cloud core and a prestellar core and we are possibly witnessing for the first time the different steps of the quasi-static contraction. Finally, the main dust peak is $30^{\prime \prime}$ off its related molecular peak, thus suggesting strong depletion of all observed molecular species in this core. This subject will be developed in the next paper (III. The gas depletion).

Acknowledgements. J.R. Pardo acknowledges support for his work from Spanish MCyT grant AYA 2000-1784, ESP 2002-01627, AYA 2002-10113-E. We thank S. Cabrit for fruitful discussions and help. Thanks to M. Dantel-Fort who performed the CFHT12K image data reduction. This publication makes use of data products from the Two Micron All Sky Survey, which is a joint project of the University of Massachusetts and the Infrared Processing and Analysis Center/California Institute of Technology, funded by the National Aeronautics and Space Administration and the National Science Foundation. The DENIS project has been partly funded by the SCIENCE and the HCM plans of the European Commission under grants CT920791 and CT940627. It is supported by INSU, MEN and CNRS in France, by the State of Baden-Württemberg in Germany, by DGICYT in Spain, by CNR in Italy, by FFwFBWF in Austria, by FAPESP in Brazil, by OTKA grants F-4239 and F-013990 in Hungary, and by the ESO C\&EE grant A-04-046. Finally, we thank the anonymous referee for his/her suggested improvements.

\section{References}

Bacmann, A., André, P., Puget, J.-L., et al. 2000, A\&A, 361, 555

Bernard, J-P., Abergel, A., Ristorcelli, I., et al. 1999, A\&A, 347, 640

Bertin, E., \& Arnouts, S. 1996, A\&AS, 117, 393

Bohlin, R. C., Savage, B. D., \& Drake, J. F. 1978, ApJ, 224, 132

Broguière, D., Neri, R., \& Sievers, A. 1995, NIC Bolometer Users Guide (IRAM Int. Rep.)

Cambrésy, L., Boulanger, F., Lagache, G., \& Stepnik, B. 2001, A\&A, 375, 999

Cambrésy, L., Beichman, C. A., Jarrett, T. H., \& Cutri, R. M. 2002, AJ, 123, 2559

Cutri, R. M., Skrutskie, M. F., Van Dyk, S., et al. 2000, Explanatory Supplement to the 2MASS Second Incremental Data Release

Dickens, J. E., Irvine, W. M., Snell, R. L., et al. 2000, ApJ, 542, 870

Dupac, X., Bernard, J. P., Boudet, N., et al. 2003, A\&A, 404, L11

Draine, B. T., \& Lee, H. M. 1984, ApJ, 285, 89

Epchtein, N. 1997, in The Impact of Large Scale Near-Infrared Sky Surveys, ed. F. Garzón, N. Epchtein, A. Omont, B. Burton, \& P. Persi (Tenerife: Kluwer Academic Publishers), 15

Epchtein, N., Deul, E., \& Derrière, S., et al. 1999, A\&A, 349, 236

Evans, N. J. II, Rawlings, J. M. C., Shirley, Y.L., \& Mundy, L. G. 2001, ApJ, 557, 193

Franco, G. 1989, A\&A, 223, 313

Hildebrand, R. H. 1983, QJRAS, 24, 267

Juvela, M., Mattila, K., Lehtinen, K., et al. 2002, A\&A, 382, 583

Lada, C. J., Lada, E. A., Clemens, D. P., \& Bally, J. 1994, ApJ, 429, 694

Lada, C. J., Alves, J., \& Lada, E. A. 1999, ApJ, 512, 250

Laureijs, R. J., Clark, S. O., \& Prusti, T. 1991, ApJ, 371, 602 
Laureijs, R. J., Fukui, Y., Helou, G., et al. 1995, ApJS, 101, 87 Lehtinen, K., Mattila, K., Lemke, D., et al. 2003, A\&A, 398, 571 Mathis, J. S. 1990, ARA\&A, 28, 37

Miville-Deschênes, M.-A., Boulanger, F., Abergel, A. \& Bernard, J.-P. 2000, A\&AS, 146, 519

McLaughlin, D. E., \& Pudritz, R. E. 1996, ApJ, 469, 194

McLaughlin, D. E., \& Pudritz, R. E. 1997, ApJ, 476, 750

Motte, F., André, P., \& Neri, R. 1998, A\&A, 336, 150

Ossenkopf, V. 1993, A\&A, 280, 617

Pagani, L., Pardo, J.-R., Fich, M., Motte, F., \& Stepnik, B. 2002, in Infrared and Submillimeter Space Astronomy, EAS Series, Vol. 4, held in Toulouse, June 2001, ed. M. Giard et al. (Les Ulis: EDP Sciences), 145

Pagani, L., Pardo, J.-R., Cambrésy, L., et al., 2003a, in Chemistry as a Diagnostic of Star Formation, held in Waterloo, Canada, Aug. 2002, ed. C. L. Curry, \& M. Fich, in press
Pagani, L., Lagache, G., Bacmann, A., et al. 2003b, A\&A, 406, L59 (Paper I)

Rengarajan, T. N. 1984, A\&A, 140, 213

Rieke, G. H., \& Lebofsky, M. J. 1985, ApJ, 288, 618

Shu, F. H. 1977, ApJ, 214, 488

Stamatellos, D., \& Whitworth, A. P. 2003, A\&A, 407, 941

Stepnik, B., Abergel, A., Bernard, J.-P., et al. 2003, A\&A, 398, 551

Tafalla, M., Mardones, D., Myers, P. C., et al. 1998, ApJ, 504, 900

Ward-Thompson, D., Scott, P. F., Hills, R. E., \& André, P. 1994, MNRAS, 268, 276

Ward-Thompson, D., Kirk, J. M., Crutcher, R. M., et al. 2000, ApJ, 537, L135

Ward-Thompson, D., André, P., Kirk, J. M. 2002, MNRAS, 329, 257

Williams, J. P., Myers, P. C., Wilner, D. J., \& di Francesco, J. 1999, ApJ 513, L61

Zucconi, A., Walmsley, C. M., Galli, D. 2001, A\&A, 376, 650 